VoL. 60 (1999) [303-318]

\title{
THE IDEAL STRUCTURE OF NILPOTENT-GENERATED TRANSFORMATION SEMIGROUPS
}

\author{
M. Paula O. Marques-Smith and R.P. Sullivan
}

To Bernhard Neumann with respect and gratitude

\begin{abstract}
In 1987 Sullivan determined the elements of the semigroup $N(X)$ generated by all nilpotent partial transformations of an infinite set $X$; and later in 1997 he studied subsemigroups of $N(X)$ defined by restricting the index of the nilpotents and the cardinality of the set. Here, we describe the ideals and Green's relations on such semigroups, like Reynolds and Sullivan did in 1985 for the semigroup generated by all idempotent total transformations of $X$. We then use this information to describe the congruences on certain Rees factor semigroups and to construct families of congruence-free semigroups with interesting algebraic properties. We also study analogous questions for $X$ finite and for one-to-one partial transformations.
\end{abstract}

\section{INTRODUCTION}

In [12] the authors described the ideals of the semigroup $E(X)$ generated by all proper (that is, non-identity) idempotent total transformations of an infinite set $X$. Given that the semigroup $P(X)$ of all partial transformations of $X$ contains nilpotents (as well as idempotents) and that the semigroup $N(X)$ generated by all nilpotents in $P(X)$ has been determined by Sullivan [13], an obvious problem is to describe the ideal structure of $N(X)$.

In this paper, we solve that problem for $X$ finite and infinite, and do the same for some natural subsemigroups of $N(X)$ : for example, the semigroup $N(X, 2)$ generated by all nilpotents of $P(X)$ with index 2 . We also characterise Green's relations on such semigroups and produce a new class of congruence-free 0 -bisimple semigroups. In doing all this, we also consider analogous questions for the nilpotent-generated subsemigroup of $I(X)$, the symmetric inverse semigroup on $X$.

\section{Received 9th January, 1999}

The second author gratefully acknowledges the generous support of Centro de Matematica, Universidade do Minho, and an Australian Research Commission Small Grant, during his visit in November 1998.

Copyright Clearance Centre, Inc. Serial-fee code: 0004-9727/99 \$A2.00+0.00. 


\section{INFINITE SETS}

In this section, $X$ will be an infinite set with cardinal $k$, and if $n$ is any infinite cardinal then $n^{\prime}$ will denote the successor of $n$ (that is, the least cardinal greater than $n)$.

All notation and terminology will be from [1] and [5] unless specified otherwise. In particular, $P(X)$ denotes the semigroup under composition of all partial transformations of $X$ (that is, all transformations $\alpha$ whose domain, $\operatorname{dom} \alpha$, is a subset of $X$ ). Note that $P(X)$ contains a zero (namely, the empty mapping $\emptyset$ ): we say $\alpha \in P(X)$ is nilpotent with index $r$ if $\alpha^{r}=\emptyset$ and $\alpha^{r-1} \neq \emptyset$.

If $\alpha \in P(X)$, we let $r(\alpha)$ denote the rank of $\alpha$ (that is, $|X \alpha|)$ and put

$$
\begin{array}{ll}
D(\alpha)=X \backslash X \alpha, & d(\alpha)=|D(\alpha)|, \\
G(\alpha)=X \backslash \operatorname{dom} \alpha, & g(\alpha)=|G(\alpha)| .
\end{array}
$$

The cardinal numbers $d(\alpha)$ and $g(\alpha)$ are called the defect and the gap of $\alpha$ and were used by Sullivan [13] to characterise the elements of $P(X)$ that can be written as a product of nilpotents in $P(X)$. For convenience we state his main results for infinite $X$ in the following two Theorems.

However, before doing that, recall that a cardinal $k$ is regular if $\left|\bigcup\left\{A_{i}: i \in I\right\}\right|=k$ implies either $|I|=k$ or some $A_{i}$ has cardinal $k$; and $k$ is singular if it is not regular. Also, if $m$ is any infinite cardinal then $\mathrm{cf}(m)$, the cofinality of $m$, is the least cardinal $n$ such that $m$ can be expressed as a sum of $n$ cardinals each less than $m$. Hence, cf $(m) \leqslant m$, and equality occurs if and only if $m$ is regular. Finally, we say $\alpha \in P(X)$ is spread over its rank if for each cardinal $p<r(\alpha)$, there exists $y \in X$ with $\left|y \alpha^{-1}\right|>p$.

The following two results summarise [13, Corollary 3 and Theorem 4$]$ and $[15$, Lemmas 2.5 and 3.2].

THEOREM 1. Let $k$ be regular and $\alpha \in P(X)$. Then $\alpha \in N(X)$ if and only if $g(\alpha) \neq 0, d(\alpha)=k$ and $g(\alpha)=k$ or $\left|y \alpha^{-1}\right|=k$ for some $y \in X$. Moreover, when this occurs, $N(X)$ is a regular semigroup and each $\alpha \in N(X)$ is a product of three or fewer nilpotents with index at most 3 .

TheOREM 2. Let $k$ be singular and $\alpha \in P(X)$. Then $\alpha \in N(X)$ if and only if $g(\alpha) \neq 0, d(\alpha)=k$ and either $g(\alpha) \geqslant r(\alpha)$ or $\alpha$ is spread over its rank. Moreover, when this occurs, $N(X)$ is a regular semigroup and each $\alpha \in N(X)$ is a product of four or fewer nilpotents with index at most 4.

While showing in [15] that the numbers 3 and 4 above are best possible, Sullivan considered another two nilpotent-generated semigroups: namely, $N(X, 2)$ generated by all nilpotents in $P(X)$ with index 2 , and $N(X, 3)$ generated by all nilpotents in $P(X)$ with index at most 3 . The following two results are [15, Theorems $2.3,3.3$ and 5.1]. 
THEOREM 3. Let $k$ be an arbitrary infinite cardinal and $\alpha \in P(X)$. Then $\alpha$ is a product of nilpotents in $P(X)$ with index 2 if and only if $d(\alpha)=k$ and $g(\alpha) \geqslant r(\alpha)$. Moreover, when this occurs, $N(X, 2)$ is a regular semigroup and each $\alpha \in N(X, 2)$ is a product of three or fewer nilpotents with index 2.

THEOREM 4. Let $k$ be singular and $\alpha \in P(X)$. Then $\alpha$ is a product of nilpotents in $P(X)$ with index at most 3 if and only if $g(\alpha) \neq 0, d(\alpha)=k$ and

(a) $g(\alpha) \geqslant r(\alpha)$, or

(b) $\left|y \alpha^{-1}\right| \geqslant r(\alpha)$ for some $y \in X$, or

(c) $g(\alpha) \geqslant \operatorname{cf}(k)$ and $\alpha$ is spread over its rank.

Moreover, when this occurs, $N(X, 3)$ is a regular semigroup and each $\alpha \in N(X, 3)$ is a product of three or fewer nilpotents with index at most 3.

As usual, we let $I(X)$ denote the symmetric inverse semigroup on $X$ and recall a theorem describing the semigroup $N I(X)$ generated by the nilpotents of $I(X)$ (see [13, Corollary 4]).

THEOREM 5. Let $k$ be an arbitrary infinite cardinal and $\alpha \in I(X)$. Then $\alpha$ is a product of nilpotents in $I(X)$ if and only if $d(\alpha)=g(\alpha)=k$. Moreover, when this occurs, $N I(X)$ is an inverse semigroup and each $\alpha \in N I(X)$ is a product of three or fewer nilpotents with index 2.

Our aim in this section is to describe the ideals and Green's relations on each of the afore-mentioned semigroups:

$$
N I(X) \subset N(X, 2) \subset N(X, 3) \subseteq N(X)
$$

when $|X|=k$ is infinite. To do this, we let $1 \leqslant \xi \leqslant k^{\prime}$ and write

$$
N_{\xi}(X)=\{\alpha \in N(X): r(\alpha)<\xi\}
$$

Since $r(\alpha \beta) \leqslant \min \{r(\alpha), r(\beta)\}$ for all $\alpha, \beta \in P(X)$, each $N_{\xi}(X)$ is an ideal of $N(X)$.

In what follows, we extend the convention introduced in [1, vol. 2 , p.241]: namely, if $\alpha \in P(X)$ is non-zero then we write

$$
\alpha=\left(\begin{array}{c}
A_{i} \\
x_{i}
\end{array}\right)
$$

and take as understood that the subscript $i$ belongs to some (unmentioned) index set $I$, that the abbreviation $\left\{x_{i}\right\}$ denotes $\left\{x_{i}: i \in I\right\}$, and that $X \alpha=\left\{x_{i}\right\}, x_{i} \alpha^{-1}=A_{i}$ and $\operatorname{dom} \alpha=\bigcup\left\{A_{i}: i \in I\right\}$. 
REMark. If $\alpha \in P(X)$ is a product of idempotents then $d(\alpha) \neq 0$ and if it is a product of nilpotents then $g(\alpha) \neq 0$. In addition, if $\beta \in P(X)$ then $X \alpha \beta \subseteq X \beta$ and $\operatorname{dom} \alpha \beta \subseteq \operatorname{dom} \alpha$ imply $d(\alpha \beta) \geqslant d(\beta)$ and $g(\alpha \beta) \geqslant g(\alpha)$. Rather surprisingly, if both $\alpha$ and $\beta$ are generated by idempotent total transformations then $d(\alpha \beta) \geqslant d(\alpha)$, whereas if they are generated by nilpotent partial transformations, it is possible that $g(\alpha \beta)<g(\beta)$ : the first statement follows from [12, Theorem 1] (when $\alpha, \beta$ have finite shift) and from [6, Lemma 2.10] (when at least one of $\alpha, \beta$ has infinite shift); and for the second statement we provide the following example.

Let $X=A \cup\left\{x_{i}\right\} \cup\{y\}$ where $|A|=k \geqslant \aleph_{0}$, and write

$$
\alpha=\left(\begin{array}{cc}
A & x_{i} \\
y & x_{i}
\end{array}\right), \quad \beta=\left(\begin{array}{ll}
y & x_{i} \\
y & x_{i}
\end{array}\right)
$$

Then, by Theorems 1 and $2, \alpha, \beta \in N(X)$ and $g(\alpha \beta)=1<k=g(\beta)$.

This means that, whereas "defect" played a major role in describing the ideals of idempotent-generated transformation semigroups in [12], the corresponding problem for nilpotent-generated semigroups does not appear to depend on "gap" in the same way.

THEOREM 6. If $|X|=k$ is infinite, the ideals of $N(X)$ are precisely $N_{\xi}(X)$ for $1 \leqslant \xi \leqslant k^{\prime}$.

Proof: We follow the argument of [1, vol. 2, Theorem 10.59]. Let $I$ be an ideal of $N(X)$ and let $\xi$ be the least cardinal greater than $r(\alpha)$ for all $\alpha \in I$. Then $I \subseteq N_{\xi}(X)$ and if $\beta \in N_{\xi}(X)$, there must exist some $\alpha \in I$ with $r(\beta) \leqslant r(\alpha)$ (otherwise, we contradict the choice of $\xi$ ). Write

$$
\beta=\left(\begin{array}{c}
B_{m} \\
x_{m}
\end{array}\right), \quad \alpha=\left(\begin{array}{c}
A_{n} \\
y_{n}
\end{array}\right)
$$

and $\left\{y_{n}\right\}=\left\{y_{m}\right\} \cup\left\{y_{p}\right\}$ where $P$ is possibly empty. Choose $a_{m} \in A_{m}$ for each $m$ and let

$$
\lambda=\left(\begin{array}{c}
B_{m} \\
a_{m}
\end{array}\right), \quad \mu=\left(\begin{array}{c}
y_{m} \\
x_{m}
\end{array}\right) .
$$

By Theorems 1 and $2, d(\alpha)=k=d(\beta)$ and so $g(\mu)=k=d(\mu)$; hence, by Theorem 5 , $\mu \in N I(X)$.

Also, if $k$ is regular then $g(\alpha)=k$ or some $A_{n}$ has cardinal $k$ and, in each case, $d(\lambda)=k$. In addition, $\lambda \circ \lambda^{-1}=\beta \circ \beta^{-1}$ and hence, since $\beta \in N(X), g(\lambda) \neq 0$ and either $g(\lambda)=k$ or some $B_{m}$ has cardinal $k$. That is, $\lambda \in N(X), \beta=\lambda \alpha \mu \in I$ and therefore $N_{\xi}(X)=I$.

Suppose $k$ is singular. If $r(\alpha)=k$, we can arrange for the index set $P$ to have cardinal $k$, and then $d(\lambda)=\left|X \backslash\left\{a_{m}\right\}\right|=k$. On the other hand, if $r(\alpha)<k$ then 
$|M|<k$ and again $d(\lambda)=k$. Next, if $g(\beta) \geqslant r(\beta)$ then

$$
g(\lambda)=g(\beta) \geqslant r(\beta)=r(\lambda)
$$

and so $\lambda \in N(X)$. Clearly, the same conclusion holds if $\beta$ is spread over its rank, and the proof is complete.

THEOREM 7. If $|X|=k$ is infinite, the ideals of $N(X, 2)$ are precisely $N(X, 2)$ $\cap N_{\xi}(X)$ for $1 \leqslant \xi \leqslant k^{\prime}$.

PROOF: Using the same argument and notation as in the above proof, we find that $\mu \in N I(X) \subset N(X, 2)$. In addition, by Theorem $3, g(\beta) \geqslant r(\beta)$, so the argument in the last paragraph in the proof of Theorem 6 shows $d(\lambda)=k$ and $g(\lambda) \geqslant r(\lambda)$ : that is, $\lambda \in N(X, 2)$, and the result follows.

THEOREM 8. If $|X|=k$ is infinite, the ideals of $N(X, 3)$ are precisely $N(X, 3)$ $\cap N_{\xi}(X)$ for $1 \leqslant \xi \leqslant k^{\prime}$.

Proof: Again, using the same argument and notation as in the proof of Theorem 6, we find that $\mu \in N I(X) \subset N(X, 3)$. In addition, since $\lambda \circ \lambda^{-1}=\beta \circ \beta^{-1}$, if $\beta$ satisfies one of the conditions in Theorem 4 then $\lambda$ does also. Hence, $\lambda \in N(X, 3)$, and the result follows.

In a similar way, using Theorem 5 , we obtain the following result.

THEOREM 9. If $|X|=k$ is infinite, the ideals of $N I(X)$ are precisely $N I(X)$ $\cap N_{\xi}(X)$ for $1 \leqslant \xi \leqslant k^{\prime}$.

We now describe Green's relations on each of the semigroups in $\left(^{*}\right)$. But first we recall T.E. Hall's Theorem [5, Proposition II.4.5]: namely, if $S$ is a regular subsemigroup of a semigroup $T$ then the $\mathcal{L}$ and $\mathcal{R}$ relations on $S$ are the restrictions to $S$ of the corresponding ones on $T$. Since each of the semigroups in $\left({ }^{*}\right)$ is regular, and the $\mathcal{L}$ and $\mathcal{R}$ relations on $P(X)$ are well-known [2], we restrict our attention in what follows to $\mathcal{D}$ alone. However, before doing that, we prove the following result for comparison with [12, Theorems 5 and 6$]$.

THEOREM 10. If $X$ is an arbitrary set and $\alpha, \beta \in P(X)$ then

(a) $\beta=\lambda \alpha$ for some $\lambda \in P(X)$ if and only if $X \beta \subseteq X \alpha$,

(b) $\beta=\alpha \mu$ for some $\mu \in P(X)$ if and only if $\operatorname{dom} \beta \subseteq \operatorname{dom} \alpha$ and

$$
\left(\alpha \circ \alpha^{-1}\right) \cap(\operatorname{dom} \alpha \times \operatorname{dom} \beta) \subseteq \beta \circ \beta^{-1},
$$

(c) $\beta=\lambda \alpha \mu$ for some $\lambda, \mu \in P(X)$ if and only if $r(\beta) \leqslant r(\alpha)$,

(d) $\mathcal{D}=\mathcal{J}$. 
Proof: The proof of (a), (c) and (d) is similar to the corresponding statement for $T(X)[1$, vol. 1 , pp 52-53], so we omit the details. For (b), note that $\beta=\alpha \mu$ implies $\operatorname{dom} \beta \subseteq \operatorname{dom} \alpha$. Also, if

$$
(u, v) \in\left(\alpha \circ \alpha^{-1}\right) \cap(\operatorname{dom} \alpha \times \operatorname{dom} \beta)
$$

then $u \alpha=v \alpha$ and $v \in \operatorname{dom} \beta$ imply that $(v \alpha) \mu \neq \emptyset$ and $u \in \operatorname{dom} \beta$, so $(u, v) \in \beta \circ \beta^{-1}$. Conversely, if the condition holds, every $\beta \circ \beta^{-1}$-class is a union of some $\alpha \circ \alpha^{-1}$-classes.

To see this, write

$$
\beta=\left(\begin{array}{c}
B_{m} \\
x_{m}
\end{array}\right), \quad \alpha=\left(\begin{array}{c}
A_{n} \\
y_{n}
\end{array}\right)
$$

and, for each $m$, let

$$
N_{m}=\left\{n \in N: B_{m} \cap A_{n} \neq \emptyset\right\}
$$

Note that $N_{m} \neq \emptyset$ since $\operatorname{dom} \beta \subseteq \operatorname{dom} \alpha$. Now $B_{m} \subseteq \bigcup\left\{A_{n}: n \in N_{m}\right\}$ and we assert equality holds. For, if $y \in B_{m} \cap A_{n}$ and $x \in A_{n}$ then $x \alpha=y \alpha$ and $(x, y) \in$ $\operatorname{dom} \alpha \times \operatorname{dom} \beta$, so the condition implies $x \beta=y \beta$. Hence, $x \in B_{m}$ and the assertion follows.

We now rewrite $\alpha$ as

$$
\alpha=\left(\begin{array}{cc}
A_{m n} & A_{p} \\
y_{m n} & y_{p}
\end{array}\right)
$$

where $\left\{A_{m n}\right\}=\left\{A_{n}: n \in N_{m}\right\}$ and $\left\{y_{p}\right\}=\left\{y_{n}\right\} \backslash\left\{y_{m n}\right\}$, and define $\mu \in P(X)$ as follows:

$$
\mu=\left(\begin{array}{c}
\left\{y_{m n}\right\} \\
x_{m n}
\end{array}\right)
$$

Then, since $B_{m}=\bigcup\left\{A_{n}: n \in N_{m}\right\}$, we have $\beta=\alpha \mu$ as required.

The next result is surprising, especially when compared with the description of $\mathcal{D}$ for certain idempotent-generated transformation semigroups (see [12, Theorems 5 and 6]).

THEOREM 11. If $|X|=k$ is infinite and $\alpha, \beta \in N(X)$ then $\beta=\lambda \alpha \mu$ for some $\lambda, \mu \in N(X)$ if and only if $r(\beta) \leqslant r(\alpha)$. Hence, $\mathcal{D}=\mathcal{J}$ for $N(X)$.

PROOF: If $r(\beta) \leqslant r(\alpha)$, an argument similar to that in the proof of Theorem 6 shows there are $\lambda, \mu \in N(X)$ with $\beta=\lambda \alpha \mu$. Since the converse is clear, we conclude that $\alpha \mathcal{J} \beta$ in $N(X)$ if and only if $r(\alpha)=r(\beta)$, and we assert the same is true for $\mathcal{D}$.

For, if $\alpha, \beta \in N(X)$ and $r(\alpha)=r(\beta)$, we can write

$$
\beta=\left(\begin{array}{c}
B_{n} \\
x_{n}
\end{array}\right), \quad \alpha=\left(\begin{array}{c}
A_{n} \\
y_{n}
\end{array}\right), \quad \gamma=\left(\begin{array}{c}
B_{n} \\
y_{n}
\end{array}\right)
$$


and it is straightforward to check that $\gamma \in N(X)$. Moreover, $X \alpha=X \gamma$ implies $\alpha \mathcal{L} \gamma$, and $\beta \circ \beta^{-1}=\gamma \circ \gamma^{-1}$ implies $\gamma \mathcal{R} \beta$, so $\alpha \mathcal{D} \beta$ as required.

Since similar statements can be readily verified for each of $N I(X), N(X, 2)$ and $N(X, 3)$, we omit the details.

\section{FINITE SETS}

Sullivan described the elements of $N(X)$ for $X$ finite in a seminar at the University of St Andrews in 1984 and in a paper [13] submitted for publication in November 1984. In that work, he also studied products of nilpotents in $I(X)$ for $X$ finite, a topic that was later examined more closely by Gomes and Howie [3]. We summarise the relevant ideas as follows.

Let $X$ be finite and $|X|=n$ and write

$$
D_{n-1}=\{\alpha \in I(X): r(\alpha)=n-1\} .
$$

It is well-known that $D_{n-1}$ is a $\mathcal{D}$-class of $I(X)$. Moreover, by [3, Corollary 2.9], each nilpotent $\lambda \in D_{n-1}$ has index $n$ : that is, in the terminology of [13, p.325], $\lambda$ is an $(n-1)$-chain which means

$$
\lambda=\left(\begin{array}{cccc}
a_{1} & a_{2} & \cdots & a_{n-1} \\
a_{2} & a_{3} & \cdots & a_{n}
\end{array}\right)
$$

for some $\left\{a_{1}, \cdots, a_{n-1}\right\} \subset X$.

Note that any $\alpha \in D_{n-1}$ has a unique completion $\bar{\alpha} \in G(X)$, the symmetric group on $X$, defined by:

$$
x \bar{\alpha}= \begin{cases}x \alpha, & \text { if } x \in \operatorname{dom} \alpha, \\ b, & \text { if } x=a,\end{cases}
$$

where $X \backslash \operatorname{dom} \alpha=\{a\}$ and $X \backslash X \alpha=\{b\}$ [3, p.388]. In addition, if $\alpha, \beta \in I(X)$ and $\alpha \beta \in D_{n-1}$ then $\alpha, \beta \in D_{n-1}$ and $\overline{\alpha \beta}=\bar{\alpha} \bar{\beta}$. Hence, if

$$
E_{n-1}=\left\{\alpha \in D_{n-1}: \bar{\alpha} \text { is an even permutation }\right\}
$$

then $\alpha, \alpha \beta \in E_{n-1}$ and $\beta \in D_{n-1}$ imply $\beta \in E_{n-1}$.

Finally, we recall that the ideals of $I(X)$ are

$$
I_{r}(X)=\{\alpha \in I(X): r(\alpha)<r\}
$$

where $1 \leqslant r \leqslant n+1$. In [3, Theorem 3.18], the authors proved the following result. 
THEOREM 12. Suppose $n \geqslant 3$.

(a) If $n$ is even then $N I(X)=I_{n}(X)$.

(b) If $n$ is odd then $N I(X)=I_{n-1}(X) \cup E_{n-1}$.

Moreover, in each case, each non-zero $\alpha \in N I(X)$ is a product of $n-1$ or fewer nilpotents, each with index $n$ (and rank $n-1$ ).

The corresponding result for $P(X)$ is as follows (see [13, Theorems 1 and 2]): to state it, we let

$$
P_{r}(X)=\{\alpha \in P(X): r(\alpha)<r\}
$$

where $1 \leqslant r \leqslant n+1$.

THEOREM 13. Suppose $n \geqslant 3$ and $\alpha \in P(X)$.

(a) If $n$ is even then $\alpha \in N(X)$ if and only if $g(\alpha) \neq 0$.

(b) If $n$ is odd then $\alpha \in N(X)$ if and only if $g(\alpha) \neq 0$ and $\alpha \in P_{n-1}(X) \cup$ $E_{n-1}$.

We now determine the ideal structure of $N I(X)$ for finite $X$.

THEOREM 14. If $|X|=n \geqslant 3$, the ideals of $N I(X)$ are precisely $N I(X) \cap N_{r}(X)$ for $1 \leqslant r \leqslant n$ where $N_{r}(X)=\{\alpha \in N(X): r(\alpha)<r\}$.

Proof: As in the proof of Theorem 6, we let $I$ be a non-zero ideal of $N I(X)$ and let $r$ be the least integer greater than $r(\alpha)$ for $\alpha \in I$. If $\beta \in N I(X) \cap N_{r}(X)$, there must exist $\alpha \in I$ with $r(\beta) \leqslant r(\alpha)$ and we write

$$
\beta=\left(\begin{array}{l}
b_{p} \\
x_{p}
\end{array}\right), \quad \alpha=\left(\begin{array}{ll}
a_{p} & a_{q} \\
y_{p} & y_{q}
\end{array}\right)
$$

where $P$ and $Q$ are finite sets (the latter possibly empty). Put

$$
\lambda=\left(\begin{array}{c}
b_{p} \\
a_{p}
\end{array}\right), \quad \mu=\left(\begin{array}{c}
y_{p} \\
x_{p}
\end{array}\right)
$$

and note that if $r(\beta)<n-1$ then $\lambda, \mu$ have rank less than $n-1$ and so, by Theorem $12, \lambda, \mu \in N I(X)$. The same conclusion is valid if $r(\beta)=n-1$ and $n$ is even. Hence, we have $\beta=\lambda \alpha \mu \in I$ in these two cases.

Hence, we suppose $r(\beta)=n-1$ and $n$ is odd. In this case, $\alpha, \beta \in E_{n-1}$ and

$$
\left\{b_{1}, \cdots, b_{n-1}, u\right\}=\left\{a_{1}, \cdots, a_{n-1}, v\right\}
$$

for some $u \notin\left\{b_{1}, \cdots, b_{n-1}\right\}$ and $v \notin\left\{a_{1}, \cdots, a_{n-1}\right\}$. If $u=v$, we let $\mu$ be as before and let

$$
\lambda=\left(\begin{array}{lll}
a_{1} & \cdots & a_{n-1} \\
a_{1} & \cdots & a_{n-1}
\end{array}\right) \in E_{n-1} .
$$


If $u \neq v$ then $u=a_{n-1}$ and $v=b_{n-1}$ say, and

$$
\left\{b_{1}, \cdots, b_{n-2}\right\}=\left\{a_{1}, \cdots, a_{n-2}\right\} .
$$

In this case, we let

$$
\lambda=\left(\begin{array}{ccccc}
a_{1} & \cdots & a_{n-3} & a_{n-2} & b_{n-1} \\
a_{1} & \cdots & a_{n-3} & a_{n-1} & a_{n-2}
\end{array}\right)
$$

whose completion $\vec{\lambda}=\left(a_{n-2}, a_{n-1}, b_{n-1}\right)$ which is even. In addition, we have

$$
\alpha=\left(\begin{array}{ccccc}
a_{1} & \cdots & a_{n-3} & a_{n-1} & a_{n-2} \\
a_{1} \alpha & \cdots & a_{n-3} \alpha & a_{n-1} \alpha & a_{n-2} \alpha
\end{array}\right)
$$

and we let

$$
\mu=\left(\begin{array}{ccccc}
a_{1} \alpha & \cdots & a_{n-3} \alpha & a_{n-1} \alpha & a_{n-2} \alpha \\
a_{1} \beta & \cdots & a_{n-3} \beta & a_{n-2} \beta & b_{n-1} \beta
\end{array}\right) .
$$

Then, regardless of whether $u=v$ or $u \neq v$, we have $\beta=\lambda \alpha \mu \in E_{n-1}$ and $\lambda \alpha \in$ $E_{n-1}$. Hence, by a comment before the statement of Theorem 12 , we conclude that $\mu \in E_{n-1}$. That is, in each case, $\lambda, \mu \in N I(X)$ by Theorem 12 , and we have shown that $N I(X) \cap N_{r}(X) \subseteq I$, and equality follows.

It is now an easy matter to determine the ideals of $N(X)$ for finite $X$.

THEOREM 15. If $|X|=n \geqslant 3$ then the ideals of $N(X)$ are precisely $N(X)$ $\cap P_{r}(X)$ for $1 \leqslant r \leqslant n$.

PRoof: With the same notation as in the proof of Theorem 14, if $\beta \in N(X)$ $\cap P_{r}(X)$, there must exist $\alpha \in I$ with $r(\beta) \leqslant r(\alpha)$ and we write

$$
\beta=\left(\begin{array}{c}
B_{p} \\
x_{p}
\end{array}\right), \quad \alpha=\left(\begin{array}{cc}
A_{p} & A_{q} \\
y_{p} & y_{q}
\end{array}\right) .
$$

Then we choose $a_{p} \in A_{p}$ for each $p$ and write

$$
\lambda=\left(\begin{array}{c}
B_{p} \\
a_{p}
\end{array}\right), \quad \mu=\left(\begin{array}{c}
y_{p} \\
x_{p}
\end{array}\right) .
$$

Then $g(\lambda)=g(\beta) \neq 0$ and $g(\mu) \geqslant d(\alpha)>0$. In addition, if $r(\beta)<n-1$ then $\lambda, \mu$ have rank less than $n-1$. Hence, by Theorem 13, $\lambda, \mu \in N(X)$. The same conclusion is valid if $r(\beta)=n-1$ and $n$ is even. Also, if $r(\beta)=n-1$ and $n$ is odd then $\beta$ is $1-1$, in which case we can complete the proof as in that of Theorem 14.

We now characterise Green's relations on $N I(X)$ and $N(X)$ for finite $X$. To begin, we provide the analogue of Theorem 10 for $I(X)$ (compare [11, Lemma 1.2]): we omit the straight-forward proof. 
THEOREM 16. If $X$ is an arbitrary set and $\alpha, \beta \in I(X)$ then

(a) $\beta=\lambda \alpha$ for some $\lambda \in I(X)$ if and only if $X \beta \subseteq X \alpha$,

(b) $\beta=\alpha \mu$ for some $\mu \in I(X)$ if and only if $\operatorname{dom} \beta \subseteq \operatorname{dom} \alpha$,

(c) $\beta=\lambda \alpha \mu$ for some $\lambda, \mu \in I(X)$ if and only if $r(\beta) \leqslant r(\alpha)$,

(d) $\mathcal{D}=\mathcal{J}$.

TheOREM 17. Suppose $|X|=n$ is finite and $\alpha, \beta \in N I(X)$. Then $\beta=\lambda \alpha \mu$ for some $\lambda, \mu \in N I(X)$ if and only if $r(\beta) \leqslant r(\alpha)$. Hence, $\mathcal{D}=\mathcal{J}$ for $N I(X)$.

PROOF: If $r(\beta) \leqslant r(\alpha)$, the proof of Theorem 14 shows that there exist $\lambda, \mu \in$ $N I(X)$ with $\beta=\lambda \alpha \mu$. Since the converse is clear, we conclude that $\alpha \mathcal{J} \beta$ in $N I(X)$ if and only if $r(\alpha)=r(\beta)$, and we assert the same is true for $\mathcal{D}$.

For, if $\alpha, \beta \in N I(X)$ and $r(\alpha)=r(\beta)$, we can write

$$
\beta=\left(\begin{array}{c}
b_{p} \\
x_{p}
\end{array}\right), \quad \alpha=\left(\begin{array}{c}
a_{p} \\
y_{p}
\end{array}\right), \quad \gamma=\left(\begin{array}{c}
b_{p} \\
y_{p}
\end{array}\right)
$$

and clearly if $r(\alpha)<n-1$ then $\gamma \in N I(X)$. Moreover, by Theorem 16, we have $\alpha \mathcal{L} \gamma \mathcal{R} \beta$, so $\alpha \mathcal{D} \beta$ as required. In fact, the same is true if $r(\alpha)=n-1$ and $n$ is even.

Therefore, suppose $r(\alpha)=n-1$ and $n$ is odd. In this case, $\alpha \in E_{n-1}$ and we can follow the proof of Theorem 14 to find $\lambda \in E_{n-1}$ with $\operatorname{dom}(\lambda \alpha)=\operatorname{dom} \beta, X \lambda \alpha=X \alpha$ and $\lambda \alpha \in E_{n-1}$. Letting $\gamma=\lambda \alpha$, we have therefore shown $\alpha \mathcal{D} \beta$ in this case also. $\square$

Since a similar statement can be easily verified for $N(X)$, we omit the details.

\section{INFINITE CONGRUENCE-FREE SEMIGROUPS}

In [8, Theorem 3.2], the authors showed that if $|X|=k \geqslant \aleph_{0}$ then the semigroup

$$
T=\{\alpha \in I(X): g(\alpha)=d(\alpha)=k\}
$$

equals the subsemigroup of $I(X)$ generated by the nilpotents in $I(X)$ with index 2 , that every $\alpha \in T$ is a product of three or fewer nilpotents with index 2 , and that 3 is best possible.

Hence, $T$ is an inverse semigroup containing an ideal $I=T \cap I_{k}(X)$ and in $[8$, Theorem 4.3], the authors proved that the Rees factor semigroup $S=T / I$ is a 0 bisimple inverse semigroup generated by its nilpotents with index 2 . Moreover, after observing that

$$
\delta_{k}=\{(\alpha, \beta) \in T \times T:|\alpha \backslash \beta \cup \beta \backslash \alpha|<k\}
$$

is a congruence on $T$, they showed that $T / \delta_{k}$ is a congruence-free, 0 -bisimple, inverse semigroup generated by its nilpotents with index 2 [7, Theorem 4.10$]$. 
From what we have shown in section 2, the above work can be generalised as follows. We know from Theorem 5 above that $T=N I(X)$, the semigroup generated by all nilpotents in $I(X)$. In addition, by Theorem 9 , the ideals of $N I(X)$ are the sets

$$
M_{\xi}=\{\alpha \in N I(X): r(\alpha)<\xi\}
$$

for $1 \leqslant \xi \leqslant k^{\prime}$ and we let $Q_{\xi}(X)$ denote the Rees factor semigroup $M_{\xi^{\prime}} / M_{\xi}$. An argument similar to that in the proof of Theorem 11 shows that $Q_{\xi}(X)$ is 0 -bisimple; and it is an inverse semigroup since $\alpha^{-1} \in Q_{\xi}(X)$ for each non-zero $\alpha \in Q_{\xi}(X)$.

It is also generated by its nilpotents with index 2. For, if $\alpha \in Q_{\xi}(X)$ is non-zero then $\alpha=\lambda_{1} \cdots \lambda_{r}$ for some nilpotents $\lambda_{i} \in N I(X)$ with index 2. Since $N I(X)$ is regular and contains a zero, we can apply [9, Lemma 2.5]: that is, we can assume that each $\lambda_{i}$ has the same rank as $\alpha$ (using standard knowledge of Green's relations on $I(X)$ : see Theorem 16 above). In this case, each $\lambda_{i} \in Q_{\xi}(X)$ and $r\left(\lambda_{i}^{2}\right)=0<\xi$, so each $\lambda_{i}$ is a nilpotent in $Q_{\xi}(X)$ with index 2 .

To determine the congruences on each $Q_{\xi}(X)$, we adapt the notation and results of $\left[14\right.$, Section 3]. That is, for each $n \geqslant \aleph_{0}$, we let

$$
\begin{gathered}
D(\alpha, \beta)=\{x \in X: x \alpha \neq x \beta\}, \operatorname{dr}(\alpha, \beta)=\max (|D(\alpha, \beta) \alpha|,|D(\alpha, \beta) \beta|) \\
\Delta_{n}=\{(\alpha, \beta) \in P(X) \times P(X): \operatorname{dr}(\alpha, \beta)<n\}
\end{gathered}
$$

and note that, by [14, Theorem 3.1], each $\Delta_{n}$ is a congruence on $P(X)$. Hence, its restriction to $M_{\xi}$ is a congruence on $M_{\xi}$ for $\aleph_{0} \leqslant n \leqslant \xi$ (note that $r(\alpha), r(\beta)<\xi$ implies $\mathrm{dr}(\alpha, \beta)<\xi)$. In addition, by writing

$$
Q_{\xi}(X)=\{\alpha \in N I(X): r(\alpha)=\xi\} \cup\{0\}
$$

(or simply $Q_{\xi}$ when no confusion will arise) and letting

$$
\delta_{n}=\left[\Delta_{n} \cap\left(Q_{\xi} \times Q_{\xi}\right)\right] \cup\{(0,0)\}
$$

we find $\delta_{n}$ is a congruence on $Q_{\xi}$ (compare [14, p.567]) called the reduction of $\Delta_{n}$ to $Q_{\xi}$.

Next we recall the notation in $[14$, p.567]: namely,

$$
S(X, k, k)=\{\alpha \in I(X): r(\alpha)=d(\alpha)=g(\alpha)=k\} \cup\{0\}
$$

which equals $Q_{k}(X)$ in our present notation. The next result is [14, Corollary 3.12]: it was used in [14, pp.578-579] to deduce a major part of [8, Theorem 4.10]. 
THEOREM 18. If $|X|=k \geqslant \aleph_{0}$ then every non-trivial proper congruence on $Q_{k}(X)$ equals $\delta_{n}$ for some $n$ satisfying $\aleph_{0} \leqslant n \leqslant k$.

We now determine the congruences on $Q_{\xi}$ for $\aleph_{0} \leqslant \xi<k$.

THEOREM 19. If $|X|=k \geqslant \aleph_{0}$ then every non-trivial proper congruence $\rho$ on $Q_{\xi}(X), \aleph_{0} \leqslant \xi<k$, equals $\delta_{n}$ for some $n$ satisfying $\aleph_{0} \leqslant n \leqslant \xi$.

Proof: Suppose $(\alpha, \beta) \in \rho$ and $\mathrm{dr}(\alpha, \beta)=r>0$. By definition,

$$
D(\alpha, \beta) \subseteq \operatorname{dom} \alpha \cup \operatorname{dom} \beta
$$

and, since $\alpha, \beta$ are $1-1$ and have rank $\xi$, we know $|\operatorname{dom} \alpha \cup \operatorname{dom} \beta|=\xi$. Let

$$
U=\operatorname{dom} \alpha \cup \operatorname{dom} \beta \cup X \alpha \cup X \beta
$$

and note that $U \alpha \subseteq U$ and $U \beta \subseteq U$. Since $|U|=\xi<k$, we can choose $V \subseteq X \backslash U$ with $|V|=\xi$. Then $V$ lies entirely outside the domains and ranges of both $\alpha \mid U$ and $\beta \mid U$. Hence, if $Y=U \cup V$ then $\alpha^{*}=\alpha \mid Y$ and $\beta^{*}=\beta \mid Y$ are 1-1 partial transformations of $Y$ with gap and defect equal to $\xi$.

We now adapt an idea of [1, vol. 2, pp 244-247], and define a relation $\rho_{Y}$ on $Q_{\xi}(Y)$ as follows: $(\lambda, \mu) \in \rho_{Y}$ if and only if $(\lambda, \mu)=\left(\delta^{*}, \varepsilon^{*}\right)$ for some $(\delta, \varepsilon) \in \rho$ where $\delta^{*}, \varepsilon^{*} \in Q_{\xi}(Y)$. It is easy to check that $\rho_{Y}$ is a congruence on $Q_{\xi}(Y)$ and, by assumption, there exists $\left(\alpha^{*}, \beta^{*}\right) \in \rho_{Y}$ with $\operatorname{dr}\left(\alpha^{*}, \beta^{*}\right)=\operatorname{dr}(\alpha, \beta)=r>0$. Hence, by Theorem $18, \rho_{Y}=\delta_{n}$ for some $n$ satisfying $\aleph_{0} \leqslant n \leqslant \xi$. This means in particular that all $\alpha, \beta \in Q_{\xi}(X)$ which map $Y$ into itself and differ in finitely many places, are $\rho$-equivalent.

Thus, if $m$ is the least cardinal greater than $\operatorname{dr}(\alpha, \beta)$ where $(\alpha, \beta) \in \rho$ then $m$ is infinite. Let $(\alpha, \beta) \in \delta_{m}$ on $Q_{\xi}(X)$ and suppose $\operatorname{dr}(\alpha, \beta)=p$ (finite or infinite). If $\operatorname{dr}(\lambda, \mu)<p$ for all $(\lambda, \mu) \in \rho$, we contradict the definition of $m$. Hence, there exists $(\lambda, \mu) \in \rho$ with $p \leqslant \mathrm{~d} r(\lambda, \mu) \leqslant \xi$. In this event, we can repeat our argument at the start to show that there is a set $Z$ with cardinal $\xi$ and a congruence $\rho_{Z}$ on $Q_{\xi}(Z)$ containing a pair of elements $\alpha, \beta$ with $\operatorname{dr}(\alpha, \beta) \geqslant p$. By Theorem 18, this means that all elements of $Q_{\xi}(Z)$ which differ in $p$ places are $\rho_{Z}$-equivalent. In particular, if $Z=\left\{a_{i}\right\} \cup\left\{b_{i}\right\} \cup\left\{c_{r}\right\} \cup\left\{d_{r}\right\}$ is a disjoint union where $|I|=p$ and $|R|=\xi$ then

$$
\left(\begin{array}{ll}
a_{i} & c_{r} \\
b_{i} & c_{r}
\end{array}\right),\left(\begin{array}{ll}
a_{i} & c_{r} \\
a_{i} & c_{r}
\end{array}\right)
$$

are $\rho_{Z}$-equivalent. Consequently, as elements of $Q_{\xi}(X)$, these transformations are also $\rho$-equivalent and, by squaring them, we obtain:

$$
\left(\begin{array}{ll}
a_{i} & c_{r} \\
a_{i} & c_{r}
\end{array}\right) \sim\left(\begin{array}{l}
c_{r} \\
c_{r}
\end{array}\right) .
$$


If $p=\xi$, we can multiply the above equivalence on the right by $\gamma \in Q_{\xi}(X)$ with $\operatorname{dom} \gamma=\left\{a_{i}\right\} \cup\left\{c_{r}\right\}$ and $a_{i} \gamma=a_{i}, c_{r} \gamma=d_{r}$ to obtain:

$$
\left(\begin{array}{ll}
a_{i} & c_{r} \\
a_{i} & d_{r}
\end{array}\right) \sim\left(\begin{array}{l}
c_{r} \\
d_{r}
\end{array}\right)
$$

Squaring this equivalence then shows there is a non-zero element of $Q_{\xi}(X)$ which is $\rho$-equivalent to 0 ; and, since $Q_{\xi}(X)$ is 0 -simple, this means $\rho$ is universal.

If $p<\xi$ and $\lambda, \mu \in Q_{\xi}(X)$ with $\operatorname{dr}(\lambda, \mu)=p$, write $D=D(\lambda, \mu),\left\{x_{i}\right\}=$ $D \lambda,\left\{y_{i}\right\}=D \mu, u_{i}=x_{i} \lambda^{-1}, v_{i}=y_{i} \mu^{-1}$ and $\left\{w_{r}\right\}=(\operatorname{dom} \lambda \cup \operatorname{dom} \mu) \backslash D$. Note that, as in the notation of $\left[14\right.$, p.565], this means possibly one (but not both) of $\left\{x_{i}\right\},\left\{y_{i}\right\}$ is empty. Also, from the way in which $D(\lambda, \mu)$ is defined, $w_{r} \lambda=w_{r} \mu=z_{r} \neq \emptyset$. We now let

$$
\pi_{1}=\left(\begin{array}{cc}
u_{i} & w_{r} \\
a_{i} & c_{r}
\end{array}\right), \pi_{2}=\left(\begin{array}{cc}
a_{i} & c_{r} \\
x_{i} & z_{r}
\end{array}\right) .
$$

Multiplying (1) on the left and the right by $\pi_{1}, \pi_{2}$ respectively, we obtain:

$$
\lambda \sim\left(\begin{array}{c}
w_{r} \\
z_{r}
\end{array}\right) .
$$

Clearly, we can do the same for $\mu$ to obtain:

$$
\mu \sim\left(\begin{array}{c}
w_{r} \\
z_{r}
\end{array}\right)
$$

and hence $(\lambda, \mu) \in \rho$. That is, any two elements of $Q_{\xi}(X)$ differing in $p$ places are $\rho$-equivalent. In particular, $(\alpha, \beta) \in \rho$ and we have shown $\delta_{m} \subseteq \rho$. Since $\rho \subseteq \delta_{m}$ by choice of $m$, it follows that $\rho=\delta_{m}$.

CoROLlary 1. If $|X|=k \geqslant \xi \geqslant \aleph_{0}$ then $Q_{\xi}(X) / \delta_{\xi}$ is a congruence-free, 0 bisimple, inverse semigroup which is generated by its nilpotents with index 2 .

In [7, Theorem 4.1], Howie proved that any semigroup is embeddable in a congruence-free bisimple semigroup which is generated by its idempotents. Given the above Corollary, and the fact that any inverse semigroup can be embedded in a nilpotent-generated inverse semigroup [13, Theorem 6], we present another consequence of Theorem 19: its proof follows that of [10, Theorem 5.4] which in turn owes much to Howie's ideas in [7].

COROLlary 2. Every inverse semigroup can be embedded in a congruence-free 0-bisimple inverse semigroup which is generated by its nilpotents with index 2 .

Proof: Let $S$ be an inverse semigroup and let $k$ be an infinite cardinal such that $k \geqslant|S|$. Also, let $X=S \times(Y \cup Z)$ where $Y, Z$ are disjoint sets with cardinal $k$, 
in which case $|X|=k$. For each $a \in S$, we define $\phi_{a} \in I(X)$ by letting $\operatorname{dom} \phi_{a}=$ $S a a^{-1} \times Y$ and

$$
\left(s a a^{-1}, y\right) \phi_{a}=(s a, y)
$$

where $s \in S, y \in Y$. Since the action of $\phi_{a}$ on $S$ is simply the Vagner-Preston embedding $a \rightarrow \rho_{a}$ of $S$ into $I(S)$ [5, Theorem II.1.10] the map $\Phi: a \rightarrow \phi_{a}$ embeds $S$ into $I(X)$. Moreover, since $S \times Z$ is contained in both $D\left(\phi_{a}\right)$ and $G\left(\phi_{a}\right)$, we have $d\left(\phi_{a}\right)=k=g\left(\phi_{a}\right)$ : that is, $\phi_{a} \in Q_{k}(X)$.

Next we recall the discussion in [14, pp.566-567] to the effect that, within $I(X)$ for any infinite set $X$,

$$
(\alpha, \beta) \in \Delta_{n} \text { if and only if }|\alpha \backslash \beta \cup \beta \backslash \alpha|<n .
$$

In addition, since $a \rightarrow \rho_{a}$ is $1-1$, we know that $\rho_{a} \backslash \rho_{b} \cup \rho_{b} \backslash \rho_{a} \neq \emptyset$ whenever $a \neq b$ in $S$. Moreover, if $(u, v) \in \rho_{a} \backslash \rho_{b} \cup \rho_{b} \backslash \rho_{a}$ then, for each $y \in Y$, we have:

$$
((u, y),(v, y)) \in \phi_{a} \backslash \phi_{b} \cup \phi_{b} \backslash \phi_{a}
$$

and so $\left|\phi_{a} \backslash \phi_{b} \cup \phi_{b} \backslash \phi_{a}\right|=|Y|=k$. In other words, if $a \neq b$ in $S$ then $\left(\phi_{a}, \phi_{b}\right) \notin \delta_{k}$ and hence the map

$$
S \rightarrow Q_{k}(X) / \delta_{k}, a \rightarrow\left[\phi_{a}\right]
$$

where $\left[\phi_{a}\right]$ denotes the $\delta_{k}$-class containing $\phi_{a}$, is an embedding of $S$ into a congruencefree semigroup with the stated properties.

\section{CONGRUENCES ON FINITE SEMIGROUPS}

In view of Theorem 14, we know that the ideals of $N I(X)$ are the sets

$$
M_{r}=\{\alpha \in N I(X): r(\alpha)<r\}
$$

for $1 \leqslant r \leqslant n$ and we let $Q_{r}$ denote the Rees factor semigroup $M_{r+1} / M_{r}$ for $1 \leqslant r \leqslant$ $n-1$. Note that each $Q_{r}$ is an inverse semigroup since $\alpha^{-1} \in Q_{r}$ for each non-zero $\alpha \in Q_{r}$. And an argument similar to that near the start of section 4 shows that $Q_{r}$ is generated by its nilpotents with index 2 .

Also, an argument similar to that in the proof of Theorem 17 shows that $Q_{r}$ is 0bisimple (and hence 0 -simple). Hence, by $\left[1\right.$, vol. 1, Corollary 2.56], $Q_{r}$ is completely 0 -simple. That is, $Q_{r}$ is a 'natural' example of a nilpotent-generated completely 0 simple semigroup as discussed in [4].

We now aim to show that $Q_{n-1}$ is 'almost' congruence-free for odd integers $n \geqslant 6$. 
We start by letting $n$ be odd and observe that, by Theorem $12, Q_{n-1}=E_{n-1} \cup\{0\}$ where the operation $*$ on $Q_{n-1}$ is given by:

$$
\alpha * \beta= \begin{cases}\alpha \beta & \text { if } r(\alpha \beta)=n-1, \\ 0 & \text { if } r(\alpha \beta)<n-1 .\end{cases}
$$

Next, we recall that if $\alpha \beta \in E_{n-1}$ then $\overline{\alpha \beta}=\bar{\alpha} \bar{\beta}$ : this and the argument required for Theorem 16 produces the following result.

TheOREM 20. Suppose $\alpha, \beta \in Q_{n-1}$ are non-zero. Then

(a) $\alpha \mathcal{L} \beta$ in $Q_{n-1}$ if and only if $X \alpha=X \beta$,

(b) $\alpha \mathcal{R} \beta$ in $Q_{n-1}$ if and only if $\operatorname{dom} \alpha=\operatorname{dom} \beta$.

Consequently, the non-zero $\mathcal{H}$-classes of $Q_{n-1}$ are determined by ordered pairs $(A, B)$ where $A, B \subset X$ and $|A|=|B|=n-1$. In fact, if $\alpha \in Q_{n-1}$ is non-zero and if $\operatorname{dom} \alpha=A, X \alpha=B$ then

$$
H_{\alpha}=\left\{\beta \in Q_{n-1}: \operatorname{dom} \beta=A, X \beta=B\right\} .
$$

Clearly, if $H_{\alpha}$ is a group, it contains an idempotent; and, since an idempotent transformation fixes its range, it follows that $A=B$. In this case, since $n$ is odd, $H_{\alpha}$ consists of all $1-1$ transformations $\alpha$ mapping $A$ into $A$ whose completion $\bar{\alpha}$ is even. But, since $|A|=n-1$, this can only occur if $\alpha$ is an even permutation of $A$ : that is, $H_{\alpha}$ is the alternating group on $A$. In other words, the non-zero group $\mathcal{H}$-classes of $Q_{n-1}$ are all isomorphic to $A_{n-1}$, the alternating group of degree $n-1$.

The next result produces a class of interesting finite semigroups which have exactly one congruence other than the identity and universal congruences: that is, they are 'almost' congruence-free (compare [5, Theorem III.6.1]).

THEOREM 21. Suppose $|X|=n$ and $n$ is odd. Then $Q_{n-1}$ is a completely $0-$ simple inverse semigroup which is generated by its nilpotents with index 2 and whose non-universal congruences are in $1-1$ correspondence with the normal subgroups of $A_{n-1}$. In particular, if also $n \geqslant 6$ then $Q_{n-1}$ has exactly one non-trivial congruence that contains all the non-zero elements of $Q_{n-1}$ in one class and 0 in another class.

ProOF: We assert that the non-universal congruences on $Q_{n-1}$ are in $1-1$ correspondence with the normal subgroups of the non-zero group $\mathcal{H}$-classes of $Q_{n-1}$, hence with those of $A_{n-1}$. Since the latter is simple if $n-1 \geqslant 5$, the result then follows.

To prove the assertion, we first note that if $A_{1}, A_{2}$ are distinct subsets of $X$ with $n-1$ elements then there exists $Y \subset X$ such that the $\mathcal{H}$-class in $Q_{n-1}$ determined by $\left(A_{1}, Y\right)$ is a group and the $\mathcal{H}$-class determined by $\left(A_{2}, Y\right)$ is not a group: for example, let $Y=A_{1}$. Since the dual of this is also true (compare [1, vol. 2, Lemma 10.57]) the assertion follows as in $[1$, vol. $2, \mathrm{p} 225]$. 
Finally, we note that a finite inverse semigroup cannot always be embedded in some $Q_{n-1}$, for the simple reason that every idempotent in a completely 0-simple semigroup is primitive, and that is seldom true for an inverse semigroup.

\section{REFERENCES}

[1] A.H. Clifford and G.B. Preston, The algebraic theory of semigroups, Mathematical Surveys 7 vol 1 and 2 (American Mathematical Society, Providence, RI, 1961 and 1967).

[2] D.G. FitzGerald and G.B. Preston, 'Divisibility of binary relations', Bull. A ustral. Math. Soc. 5 (1971), 75-86.

[3] G.M.S. Gomes and J.M. Howie, 'Nilpotents in finite symmetric inverse semigroups', Proc. Edinburgh Math. Soc. 30 (1987), 383-395.

[4] C.H. Houghton and R.P. Sullivan, 'Completely zero-simple semigroups generated by nilpotent elements', Glasgow Math. J. 25 (1984), 163-165.

[5] J.M. Howie, An introduction to semigroup theory (Academic Press, London, 1976).

[6] J.M. Howie, 'Some subsemigroups of infinite full transformation semigroups', Proc. Royal Soc. Edinburgh Sect. A 88 (1981), 159-167.

[7] J.M. Howie, 'A class of bisimple, idempotent-generated congruence-free semigroups', Proc. Royal Soc. Edinburgh Sect. A 88 (1981), 169-184.

[8] J.M. Howie and M.P.O. Marques-Smith, 'Inverse semigroups generated by nilpotent transformations', Proc. Royal Soc. Edinburgh Sect. A 99 (1984), 153-162.

[9] J.M. Howie and M.P.O. Marques-Smith, 'A nilpotent-generated semigroup associated with a semigroup of full transformations', Proc. Royal Soc. Edinburgh Sect. A 108 (1988), 181-187.

[10] M.P.O. Marques, Infinite transformation semigroups, PhD Thesis (University of St. Andrews, Scotland, 1983).

[11] W.D. Munn, 'Fundamental inverse semigroups', Quart. J. Math. Oxford 21 (1970), 57-70.

[12] M.A. Reynolds and R.P. Sullivan, 'The ideal structure of idempotent-generated transformation semigroups', Proc. Edinburgh Math. Soc. 28 (1985), 319-331.

[13] R.P. Sullivan, 'Semigroups generated by nilpotent transformations', J. Algebra 110 (1987), 324-343.

[14] R.P. Sullivan, 'Congruences on transformation semigroups with fixed rank', Proc. London Math. Soc. (3) 70 (1995), 556-580.

[15] R.P. Sullivan, 'Nilpotents in semigroups of partial transformations', Bull. Austral. Math. Soc. 55 (1997), 453-467.

Centro de Matematica

Universidade do Minho

4700 Braga

Portugal

\author{
Department of Mathematics and Statistics \\ Sultan Qaboos University \\ Oman \\ and \\ Department of Mathematics \\ University of Western Australia \\ Nedlands WA 6907 \\ Australia
}

